\title{
RECURRENCES FOR THE SUM OF DIVISORS
}

\author{
JOHN A. EWELL
}

\begin{abstract}
The author presents two recursive determinations of the sum of positive divisors of a given positive integer. Each recurrence is then discussed with regard to economy of computation, and in this light is compared with the well-known recurrence of Niven and Zuckerman. As far as methods of proof are concerned, everything is accomplished within the algebra of formal power series.
\end{abstract}

For a given positive integer $n, \sigma(n)$ denotes the sum of the positive divisors of $n$; symbolically

$$
\sigma(n)=\sum_{d \mid n} d
$$

Using an argument based essentially on Euler's pentagonal-number identity, Niven and Zuckerman [2, p. 232] derive the following recursive formula for $\sigma(n)$.

THEOREM 1. For each positive integer $n$,

$$
\begin{gathered}
\sum_{k=0}(-1)^{k}\left\{\sigma\left(n-\left(3 k^{2}-k\right) / 2\right)+\sigma\left(n-\left(3 k^{2}+k\right) / 2\right)\right\} \\
= \begin{cases}(-1)^{j+1} n, & \text { if } n=\left(3 j^{2} \pm j\right) / 2, \\
0, & \text { otherwise, }\end{cases}
\end{gathered}
$$

where the sum extends as far as the arguments are positive.

In this note we derive two new recursive schemes for computing the values $\sigma(n)$. The identities on which the derivations depend are as follows:

$$
\begin{gathered}
\prod_{n=1}^{\infty}\left(1-x^{n}\right)\left(1-x^{2 n-1}\right)=1+2 \sum_{n=1}^{\infty}(-1)^{n} x^{n^{2}}, \\
\prod_{n=1}^{\infty}\left(1-x^{n}\right)^{3}=\sum_{n=0}^{\infty}(-1)^{n}(2 n+1) x^{n(n+1) / 2}
\end{gathered}
$$

For proofs see [1, pp. 282-285] where both of these identities are derived as special cases of the celebrated Gauss-Jacobi triple-product identity. To facilitate both statement and proof of the first of our two results, we introduce the following notation. Recall that every positive integer $n$ has a unique repre-

Received by the editors September 14, 1976.

AMS (MOS) subject classifications (1970). Primary 10A20; Secondary 10A35.

Key words and phrases. Recurrences, sum of the positive divisors of a positive integer, identities. 
sentation as $n=2^{b(n)} O(n)$ where $b(n)$ is a nonnegative integer and $O(n)$ is odd. Now define

$$
\omega(n)=\sigma(n)+\sigma(O(n))
$$

Note that: owing to the multiplicativity of $\sigma, \omega(n)=2^{b(n)+1} \sigma(O(n))$. We are now prepared to state our results.

THEOREM 2. For each positive integer $m$,

$$
\sigma(2 m-1)-\sum_{l=1} \omega\left(2 m-1-(2 l-1)^{2}\right)+2 \sum_{l=1} \sigma\left(2 m-1-(2 l)^{2}\right)
$$

$$
= \begin{cases}n^{2}, & \text { if } 2 m-1=n^{2} \\ 0, & \text { otherwise, }\end{cases}
$$

and

$$
\begin{gathered}
\omega(2 m)-4 \sum_{l=1} \sigma\left(2 m-(2 l-1)^{2}\right)+2 \sum_{l=1} \omega\left(2 m-(2 l)^{2}\right) \\
= \begin{cases}-2 n^{2}, & \text { if } 2 m=n^{2}, \\
0, & \text { otherwise, }\end{cases}
\end{gathered}
$$

where in both (4) and (5) summation extends as far as the arguments are positive.

THEOREM 3. For each positive integer $n$,

$$
\begin{aligned}
& \sum_{l=0}(-1)^{l}(2 l+1) \sigma\left(n-\frac{l(l+i)}{2}\right) \\
& \quad= \begin{cases}(-1)^{j+1} \frac{j(j+1)(2 j+1)}{6}, & \text { if } n=\frac{j(j+1)}{2}, \\
0, & \text { otherwise, }\end{cases}
\end{aligned}
$$

where the summation convention is as before.

Proof of Theorem 2. Briefly, set $F(x)=1+2 \Sigma(-1)^{n} x^{n^{2}}$. Now take the logarithmic derivative of identity (2) and multiply the resulting identity by $x$ to obtain

$$
\sum_{n=1}^{\infty} \frac{n x^{n}}{1-x^{n}}+\sum_{n=1}^{\infty} \frac{(2 n-1) x^{2 n-1}}{1-x^{2 n-1}}=-\frac{x F^{\prime}(x)}{F(x)} .
$$

We develop the first sum on the left of (7) to obtain

$$
\begin{aligned}
\sum_{n=1}^{\infty} \frac{n x^{n}}{1-x^{n}} & =\sum_{n=1}^{\infty} n x^{n} \sum_{j=0}^{\infty} x^{j n}=\sum_{n=1}^{\infty} \sum_{j=1}^{\infty} n x^{j n} \\
& =\sum_{k=1}^{\infty} x^{k} \sum_{n \mid k} n=\sum_{k=1}^{\infty} \sigma(k) x^{k}
\end{aligned}
$$


Development of the second sum yields

$$
\begin{aligned}
& \sum_{n=1}^{\infty} \frac{(2 n-1) x^{2 n-1}}{1-x^{2 n-1}}=\sum_{n=1}^{\infty}(2 n-1) x^{2 n-1} \sum_{j=0}^{\infty} x^{j(2 n-1)} \\
& =\sum_{n=1}^{\infty} \sum_{j=1}^{\infty}(2 n-1) x^{j(2 n-1)} \\
& =\sum_{k=1}^{\infty} x^{k} \sum_{d \mid k,} d=\sum_{m=1}^{\infty} \sigma(2 m-1) x^{2 m-1}+\sum_{m=1}^{\infty} \sigma(O(2 m)) \dot{x}^{2 m} .
\end{aligned}
$$

Thus, identity (7) becomes

$$
2 \sum_{m=1}^{\infty} \sigma(2 m-1) x^{2 m-1}+\sum_{m=1}^{\infty} \omega(2 m) x^{2 m}=-\frac{x F^{\prime}(x)}{F(x)}
$$

Now,

$$
\begin{aligned}
\left\{2 \sum_{m=1} \sigma(2 m-1) x^{2 m-1}\right\} F(x)=2 \sum_{m=1}^{\infty} \sigma(2 m-1) x^{2 m-1} \\
+\left\{2 \sum_{k=1}^{\infty} \sigma(2 k-1) x^{2 k-1}\right\}\left\{2 \sum_{l=1}^{\infty}(-1)^{l} x^{l^{2}}\right\} \\
=2 \sum_{m=1}^{\infty} \sigma(2 m-1) x^{2 m-1}+\sum_{m=1}^{\infty} x^{2 m-1} \sum_{l=1} 4 \sigma\left(2 m-1-(2 l)^{2}\right) \\
-\sum_{m=1}^{\infty} x^{2 m} \sum_{l=1} 4 \sigma\left(2 m-(2 l-1)^{2}\right) .
\end{aligned}
$$

And,

$$
\begin{aligned}
\left\{\sum_{m=1}^{\infty} \omega(2 m) x^{2 m}\right\} F(x)= & \sum_{m=1}^{\infty} \omega(2 m) x^{2 m} \\
& +\left\{\sum_{k=1}^{\infty} \omega(2 k) x^{2 k}\right\}\left\{\sum_{l=1}^{\infty} 2(-1)^{l} \cdot x^{l^{2}}\right\} \\
= & \sum_{m=1}^{\infty} \omega(2 m) x^{2 m}+\sum_{m=1}^{\infty} x^{2 m} \sum_{l=1} 2 \omega\left(2 m-(2 l)^{2}\right) \\
& -\sum_{m=1}^{\infty} x^{2 m-1} \sum_{l=1} 2 \omega\left(2 m-1-(2 l-1)^{2}\right) .
\end{aligned}
$$

Finally, in the identity

$$
\begin{gathered}
\left\{2 \sum_{m=1}^{\infty} \sigma(2 m-1) x^{2 m-1}\right\} F(x)+\left\{\sum_{n=1}^{\infty} \omega(2 m) x^{2 m}\right\} F(x) \\
=-x F^{\prime}(x)=2 \sum_{n=1}^{\infty}(-1)^{n+1} \cdot n^{2} x^{n^{2}},
\end{gathered}
$$


we equate coefficients of odd powers $x^{2 m-1}$ to obtain recurrence (4) and equate coefficients of even powers $x^{2 m}$ to obtain (5).

Proof of Theorem 3. Here we set $G(x)=\Sigma(-1)^{n}(2 n+1) x^{n(n+1) / 2}$ and operate on identity (3) to obtain

$$
3 \sum_{n=1}^{\infty} \frac{n x^{n}}{1-x^{n}}=-\frac{x G^{\prime}(x)}{G(x)},
$$

or equivalently,

$$
\left\{\sum_{n=1}^{\infty} \frac{n x^{n}}{1-x^{n}}\right\} G(x)=-\frac{1}{3} x G^{\prime}(x) .
$$

From the proof of Theorem 2 we know the expansion of the sum on the left side of the foregoing identity. Hence, we easily find the complete expansions of both sides and equate coefficients of like powers to arrive at recurrence (6).

REMARKS. It is of interest to compare the recurrences of these three theorems. If, for example, one had in mind adaptation of the recurrences to machine use, then since the machine immediately determines the binary part of given $n$ and $\sigma(n)=\left(2^{b(n)+1}-1\right) \sigma(O(n))$, one may assume that the given number $n$ is odd. And now one has only to compare recurrences (1), (4) and (6). Then, for an arbitrary choice of "large" $n$ odd, recurrence (1) needs about $2 \sqrt{\frac{2}{3} n}$ of the previous values, recurrence (4) needs about $\sqrt{n}$ of them and recurrence (6) needs about $\sqrt{2 n}$ of them. In order to fix ideas let us consider the not-too-large value of $n=63$ and partially compute $\sigma(63)$ by each of the recurrences.

By recurrence (1),

$$
\begin{aligned}
\sigma(63)= & \sigma(62)+\sigma(61)-\sigma(58)-\sigma(56)+\sigma(51)+\sigma(48) \\
& -\sigma(41)-\sigma(37)+\sigma(28)+\sigma(23)-\sigma(12)-\sigma(6) .
\end{aligned}
$$

By recurrence (4),

$$
\begin{aligned}
\sigma(63)= & 2^{2} \cdot \sigma(31)+2^{2} \cdot \sigma(27)+2^{2} \cdot \sigma(19)+2^{2} \cdot \sigma(7) \\
& -2 \cdot \sigma(59)-2 \cdot \sigma(47)-2 \cdot \sigma(27) .
\end{aligned}
$$

And, by recurrence (6),

$$
\begin{aligned}
\sigma(63)= & 3 \cdot \sigma(62)-5 \cdot \sigma(60)+7 \cdot \sigma(57)-9 \cdot \sigma(53) \\
& +11 \cdot \sigma(48)-13 \cdot \sigma(42)+15 \cdot \sigma(35)-17 \cdot \sigma(27) \\
& +19 \cdot \sigma(18)-21 \cdot \sigma(8) .
\end{aligned}
$$

Thus, formally speaking, recurrence (1) uses 12 lower values, recurrence (4) uses 7 (which reduces to 6 since $\sigma(27)$ occurs twice) and recurrence (6) uses 10. Of the three recurrences, (4) is by far the most economical. Recurrence (6) is better than (1) since computation of the additional coefficients $2 l+1$ involves no difficulty whatever. 


\section{REFERENCES}

1. G. H. Hardy and E. M. Wright, An introduction to the theory of mumbers, 4th ed., Clarendon Prese, Oxford, 1960.

2. I. Niven and H. S. Zuckerman, An introduction to the theory of numbers, 3rd ed., Wiley, New York, 1972.

Departmient of Mathimatical Sctences, Northern Ilunnols Untverstty, DeKals, Ilunvots 60115 